\title{
Spontaneous Magnetic Superdomain Wall Fluctuations in an Artificial Antiferromagnet
}

\author{
X. M. Chen, ${ }^{1,2,{ }^{*}}$ B. Farmer, ${ }^{3}$ J. S. Woods, ${ }^{3,4}$ S. Dhuey, ${ }^{5}$ W. $H u,{ }^{6}$ C. Mazzoli, ${ }^{6}$ S. B. Wilkins $\odot,{ }^{6}$ R. V. Chopdekar $\odot,{ }^{1}$ \\ A. Scholl, ${ }^{1}$ I. K. Robinson $\odot,{ }^{7,8}$ L. E. De Long $\odot,{ }^{3}$ S. Roy $\odot,{ }^{1, \dagger}$ and J. T. Hastings ${ }^{2, \$}$ \\ ${ }^{1}$ Advanced Light Source, Lawrence Berkeley National Laboratory, Berkeley, California 94720, USA \\ ${ }^{2}$ Department of Electrical and Computer Engineering, University of Kentucky, Lexington, Kentucky 40506, USA \\ ${ }^{3}$ Department of Physics and Astronomy, University of Kentucky, Lexington, Kentucky 40506, USA \\ ${ }^{4}$ Materials Science Division, Argonne National Laboratory, Lemont, Illinois 60439, USA \\ ${ }^{5}$ Molecular Foundry, Lawrence Berkeley National Laboratory, Berkeley, California 94720, USA \\ ${ }^{6}$ National Synchrotron Light Source II, Brookhaven National Laboratory, Upton, New York 11973, USA \\ ${ }^{7}$ Condensed Matter Physics and Materials Science Department, Brookhaven National Laboratory, Upton, New York 11973, USA \\ ${ }^{8}$ London Centre for Nanotechnology, University College, Gower Street, London WC1E 6BT, United Kingdom
}

(Received 27 August 2018; revised manuscript received 16 October 2019; published 6 November 2019)

Collective dynamics often play an important role in determining the stability of ground states for both naturally occurring materials and metamaterials. We studied the temperature dependent dynamics of antiferromagnetically ordered superdomains in a square artificial spin lattice using soft $\mathrm{x}$-ray photon correlation spectroscopy. We observed an exponential slowing down of superdomain wall motion below the antiferromagnetic onset temperature, similar to the behavior of typical bulk antiferromagnets. Using a continuous time random walk model we show that these superdomain walls undergo low-temperature ballistic and high-temperature diffusive motions.

DOI: 10.1103/PhysRevLett.123.197202

Naturally occurring systems with dipole magnetic interactions exhibit exotic emergent phases, such as quantum spin liquids [1,2], and novel magnetic excitations [3]. Fluctuations about equilibrium in such systems are inevitable and remain incompletely understood. Moreover, low phase transition temperatures and lack of control in engineering the energy landscapes of atomic systems pose significant challenges to understanding the fundamental physics underlying spin ice behavior. Artificially fabricated lattices mitigate these problems and have attracted increasing attention as appropriate model systems for elucidation of frustration, phase transitions, and associated dynamics [4-7].

Artificially fabricated lattices commonly consist of dipolecoupled, elongated, nanoscale segments of ferromagnetic thin films ("block spins") placed on a two-dimensional periodic lattice. The shape anisotropy of the block spins constrains their magnetization to lie along their long axis, which creates a classical analog of Ising spins. We refer to such systems as "artificial spin lattices" (ASL), which include the intensively studied artificial spin ices [8]. In particular, a 2D square ASL exhibits an antiferromagnetic ground state [5,9-14], whose simple structure serves as an ideal model system for studies of equilibrium dynamics in dipolar-coupled systems.

Previous investigations of thermally active, square ASLs indicate that a magnetic phase transition from an ordered antiferromagnetic (AFM) ground state to a disordered paramagnetic (PM) state takes place at a temperature $T_{N}$. Large AFM domains form well below $T_{N}[11,13]$. Such mesoscopic domains are referred to as "superdomains" to distinguish them from microscopic domains in the magnetic thin film [15]. When the temperature approaches $T_{N}$, the system forms contiguous regions of rapidly fluctuating block spins coexisting with AFM superdomains [See Ref. [13] and Fig. S5(a) in the Supplemental Material [16] ].

Static AFM superdomains in square ASL have been imaged using magnetic force microscopy (MFM) [5,22]. In one case, frozen thermal excitations above the AFM ground state were observed within these superdomains [5]. Lorentz transmission electron microscopy has also been used to image similar static superdomains in square ASL with topological defects [23]. On the other hand, dynamics in the square ASL have been imaged with photoemission electron microscopy (PEEM) to study fluctuations of individual block spins [13] and relaxation from ferromagnetic states [12]. However, these studies did not capture the collective fluctuations of block spins at superdomain boundaries. Moreover, these studies were limited to the PEEM time resolution of a few seconds [12,13,24,25].

Here we report the direct observation of spontaneous AFM superdomain wall nucleation, annihilation, and fluctuations in a 2D square ASL. We have used resonant coherent $\mathrm{x}$-ray diffraction over a wide range of temperatures near the AFM-to-PM phase transition. Coherent $\mathrm{x}$ rays can directly probe order parameters and collective dynamics. The diffraction pattern of coherent $\mathrm{x}$ rays from magnetic domains includes a complex interference (speckle) pattern that is unique to the real-space superdomain textures. 

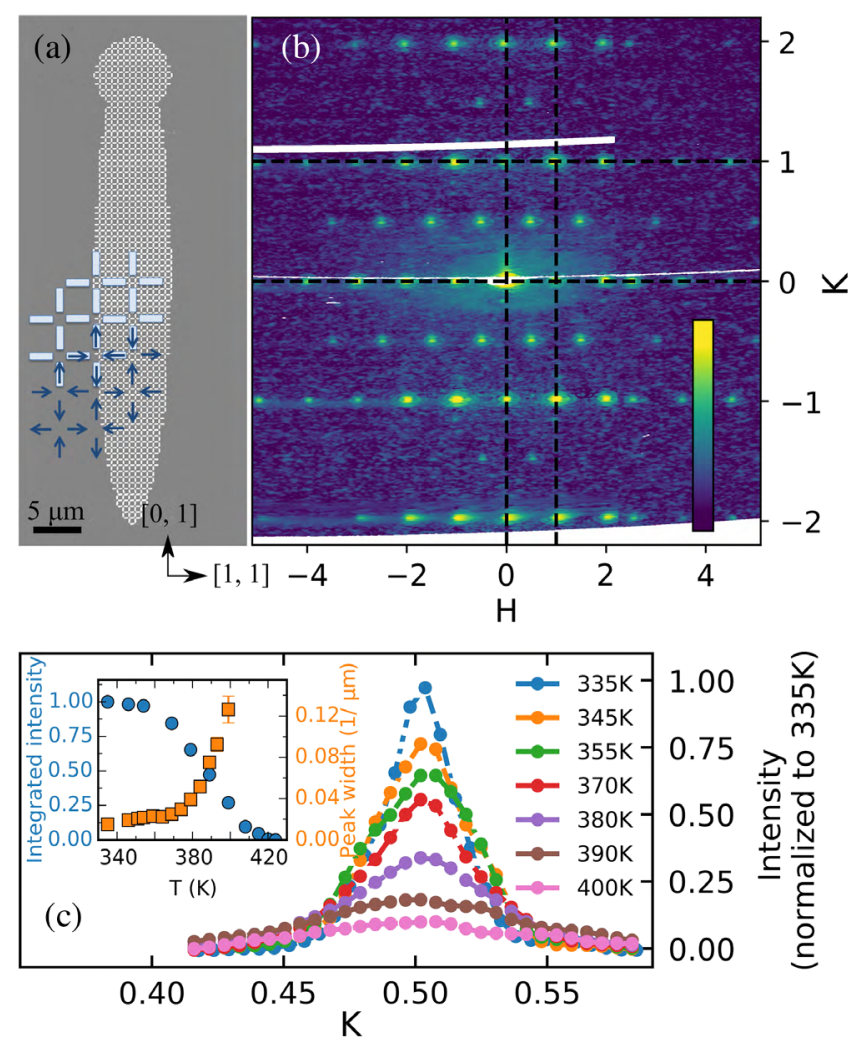

FIG. 1. (a) SEM image of a square ASL sample with a schematic of the block-spin lattice and AFM-ordered block spins. (b) ASL diffraction pattern $(T=335 \mathrm{~K})$ in reciprocal lattice coordinates $(H, K)=\left(Q_{x} /(2 \pi / a), Q_{y} /(2 \pi / b)\right)$, where $a=b=$ lattice constant $(600 \mathrm{~nm})$. Detector intensity is plotted on a log scale in arbitrary units (note color scale). A half-integer AFM Bragg peak is clearly visible at the center of the box bounded by black dotted lines. (c) Temperature dependence of detector intensity along a cut through time-averaged AFM Bragg peak. Inset shows the integrated intensity and peak width obtained from a Lorentzian fit.

By tracking time-dependent speckle motion, we studied superdomain dynamics in square ASL with 100 -ms time resolution. We applied a random-walk model that revealed two distinct regimes of superdomain wall motion as the sample goes through the AFM phase transition: a lowtemperature ballistic and a high-temperature diffusive types. These studies show that characterizing superdomain wall behavior is critical to understanding the dynamics of the square ASL. Such an understanding may prove crucial for implementing computing and data storage strategies based upon artificial spin systems [26-29].

A square permalloy $\left(\mathrm{Ni}_{0.8} \mathrm{Fe}_{0.2}\right)$ ASL was fabricated on a silicon nitride membrane using electron-beam lithography [Fig. 1(a)]. The block-spin dimensions were $470 \mathrm{~nm}$ long, $170 \mathrm{~nm}$ wide, and $3 \mathrm{~nm}$ thick with a lattice constant $a=600 \mathrm{~nm}$. Figure 1(a) also illustrates the AFM ground state configuration of the ASL. Coherent x-ray diffraction measurements were performed at beam line 12.0.2 at the Advanced Light Source and beam line 23-ID-1 at the
National Synchrotron Light Source II. The sample was positioned at a glancing angle of $\theta=10^{\circ}$ with respect to the $\sigma$-polarized beam propagation direction to enhance the inplane $\mathrm{x}$-ray magnetic cross section with the $[0,1]$ axis in the scattering plane. The detector was centered on the specularly (zero order) reflected beam. The sample's elongated shape $(\sim 8 \mu \mathrm{m} \times 50 \mu \mathrm{m})$, tailored for the $10^{\circ}$ glancing angle, maximizes the scattering volume while satisfying the Nyquist sampling condition. Essentially perfect transverse coherence and a longitudinal coherence length of $\sim 2 \mu \mathrm{m}$ are realized at the sample. The incident $\mathrm{x}$-ray beam always overfills the sample area to minimize artifacts from beam drift or from shifts in the sample position due to temperature changes. Diffracted photons were collected using a fast CCD detector (with a readout rate of $10 \mathrm{~Hz}$ and $30 \mu \mathrm{m} \times 30 \mu \mathrm{m}$ pixel size) placed $340 \mathrm{~mm}$ from the sample [30].

Figure 1(b) shows a typical diffraction pattern from a square ASL in its AFM ground state. Rows of intense structural Bragg peaks and weaker AFM Bragg peaks are visible at integer $\boldsymbol{q}=(H, K)$ and half-integer $(H / 2, K / 2)$ wave vectors, respectively, surrounding the central $(0,0)$ specular reflection. The AFM Bragg peaks could only be detected by using resonant enhancement from the magnetic Fe (Ni) edge at 707 (853) eV [16]. These peaks provide a direct measure of the strength and character of the AFM order. Figure 1(c) shows cuts through time-averaged AFM Bragg peaks at various temperatures spanning the magnetic transition. The inset plots the temperature dependence of the integrated AFM Bragg peak intensity that corresponds to the area fraction of AFM domains. The width of the AFM Bragg peak measures the AFM correlation length; thus, we observe the AFM superdomains shrink as temperature approaches the magnetic transition at $T_{N} \sim 425 \mathrm{~K}$. Past theoretical studies $[17,31]$ on square ASL predict a continuous phase transition; here, our observed transition is apparently broadened by a finite size effect $[16,32]$.

The AFM Bragg peaks show speckle patterns that arise from coherent interference between different AFM superdomains [Fig. 2(a)]. This pattern reflects the square of the Fourier transform of the AFM texture, and offers unique insights into the spatial character and dynamics of the AFM state. The size and shape of speckles depend on the $\mathrm{x}$-ray energy, sample illuminated area, and the scattering geometry [33]. However, the number of speckles and spatial distribution of intensities are an indication of the number of AFM superdomains and their dynamics [34]. After initially heating above $T_{N}$ and then cooling until $T \ll T_{N}$, only a single speckle was observed in the AFM peaks, consistent with the presence of only a single AFM superdomain across the entire sample [35]. As we increase the temperature, thermal excitations nucleate superdomain walls that split a single speckle to multiple speckles. We note that single block-spin flips or multiple block-spin excitations [5] cannot create these speckle patterns that necessarily 

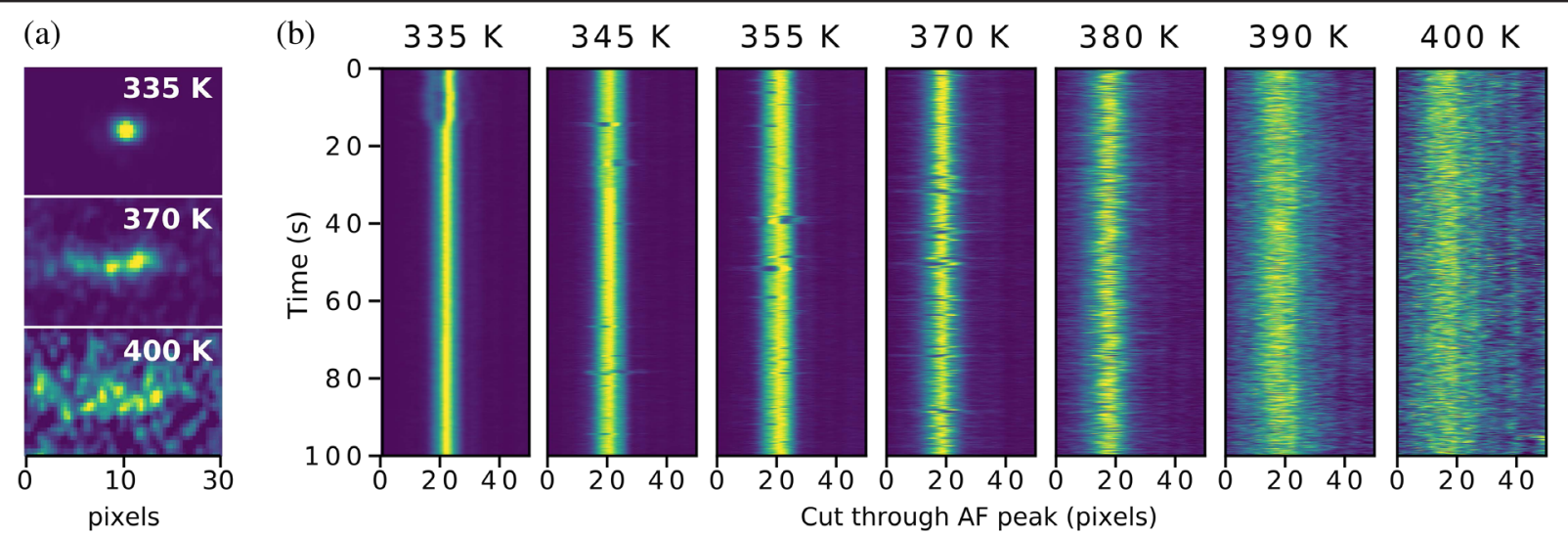

FIG. 2. (a) Speckles observed for a single AFM Bragg peak at three different temperatures. At $335 \mathrm{~K}$ we observe a single AFM superdomain, and growth of speckle number with increasing temperature. (b) Waterfall plots showing the time evolution of speckle positions for various temperatures. Each horizontal line represents a cut through an AFM peak capturing the intensity vs pixel position at some time $t$. One pixel is approximately $0.005 \mathrm{in} \mathrm{K}$. Intensities are normalized to the maximum intensity for each temperature as given in Fig. 1(c). Spontaneous domain wall fluctuations are observed at all temperatures, but decrease in number with reduced temperature.

require multiple AFM superdomains with distinct, extended boundaries.

To visualize the time evolution of speckle positions, we show "waterfall plots" in Fig. 2(b), which consist of speckle intensity measured along a vertical cut through the AFM peak as a function of time with a resolution of $0.1 \mathrm{~s}$. The fluctuation rate and number of speckles increases as the phase transition temperature is approached from below. This is a direct indication of the sample transitioning from a stable, single superdomain state to a highly fluctuating, multidomain state. Spontaneous nucleation and annihilation of superdomain walls is apparent in the data shown in Fig. 2(b). For example, at $345 \mathrm{~K}$ the system is initially in a single superdomain state that generates a single speckle. Around $t=15 \mathrm{~s}$, the speckle splits into two, indicating a creation of a superdomain wall. Subsequently, at $t=16 \mathrm{~s}$, the system evolves back to a single superdomain. This collective behavior was not considered in prior PEEM studies that focused on single block-spin fluctuations [13] and XPCS studies that focused on weakly interacting block spins [36]. Such spontaneous behavior clearly arises from an equilibrium fluctuation instead of the magnetic relaxation processes previously observed by PEEM [12].

The speckle time dependence is quantified using the onetime correlation function, $g_{2}(\boldsymbol{q}, \tau)$, given by

$$
g_{2}(\boldsymbol{q}, \tau)=\frac{\langle I(\boldsymbol{q}, t) I(\boldsymbol{q}, t+\tau)\rangle}{\langle I(\boldsymbol{q}, t)\rangle^{2}}=1+\beta|F(\boldsymbol{q}, \tau)|^{2},
$$

where $I(\boldsymbol{q}, t)$ is the total intensity of a speckle image at wave vector $\boldsymbol{q}$ and at time $t$. The brackets \langle\rangle indicate the time and ensemble average over all speckles with equivalent $\boldsymbol{q}$ values. We can further write $g_{2}(\boldsymbol{q}, \tau)$ in terms of the intermediate scattering function $|F(\boldsymbol{q}, \tau)|^{2}$ of the sample, and speckle contrast $\beta$ that depends only on the experimental setup [37,38]. We calculate $|F(\tau)|^{2}$ using detector areas corresponding to a single speckle. Figure 3(a) shows that the decay time clearly decreases with increasing temperature. Above $385 \mathrm{~K},|F(\tau)|^{2}$ flattens [16] as the fluctuations become faster than the CCD acquisition rate. We did not observe a clear $\boldsymbol{q}$ dependence of the speckle correlation up to $375 \mathrm{~K}$, as the speckle intensity drops sharply with increasing $\boldsymbol{q}$. In principle, $|F(\tau)|^{2}$ drops from 1 to 0 upon complete decorrelation of a speckle pattern. In our case, $|F(\tau)|^{2}$ drops to a temperature-dependent, finite offset that depends on the static fraction of AFM superdomains.

The dramatic temperature dependence in the curvature of $|F(\tau)|^{2}$ indicates a change in the nature of superdomain

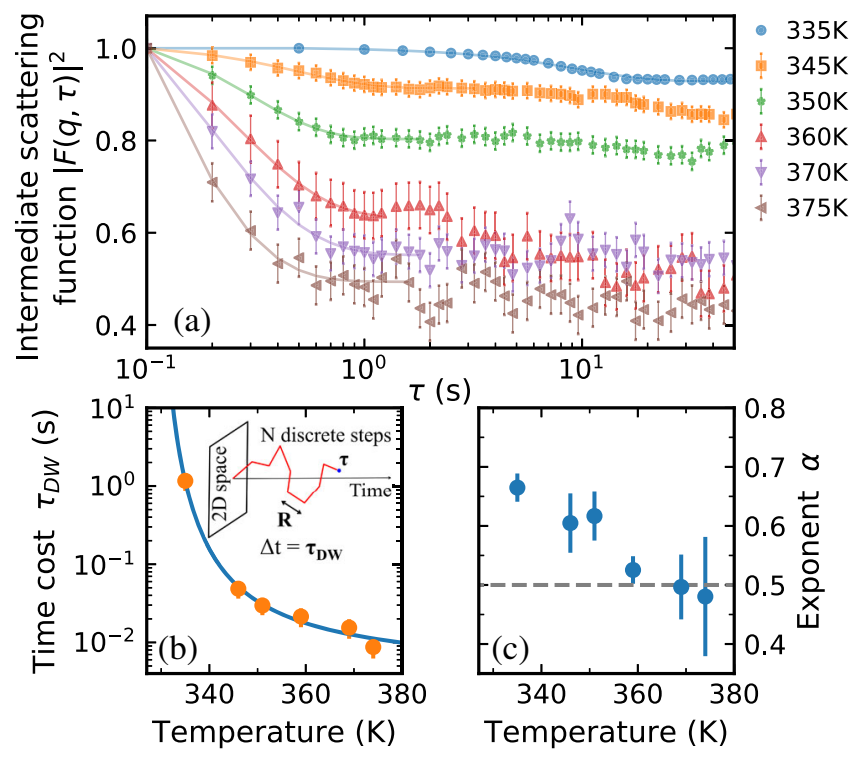

FIG. 3. (a) Intermediate scattering function $|F|^{2}$ calculated for speckle patterns at different temperatures. Solid lines are random walk fits to the initial decay. Temperature dependence of (b) time cost, $\tau_{\mathrm{DW}}$, and (c) exponent $\alpha$ in the model. [Inset in (b)] Random walk model schematic. 
dynamics. To understand this behavior, we developed a model that maps magnetic superdomains onto particles positioned at the center of mass of the superdomain boundaries [16]. In this approach, we are not sensitive to fluctuations of individual block spins, but our model adequately describes $|F(\tau)|^{2}$ because our signal is dominated by speckles in low- $q$ regions. Movements of dilute particles in media are often modeled with continuous time random walk (CTRW) behavior $[18,39,40]$ where the decorrelation of speckles at $(q, \tau)$ is the expected value of the degree of correlation $h$ weighted by its probability density function (PDF) $P_{\tau_{\mathrm{DW}}}$, such that

$$
F(q, \tau)=\sum_{N=0}^{\infty} P_{\tau_{\mathrm{DW}}}(\tau, N) h(q, N) .
$$

We take $P_{\tau_{\mathrm{DW}}}(\tau, N)$ to be a Poisson distribution $\left(\tau / \tau_{\mathrm{DW}}\right)^{N} e^{-\tau / \tau_{\mathrm{DW}}} / N$ ! describing the probability density of the number of steps $N$ that a particle traveled in time $\tau$, with variable time cost $\tau_{\mathrm{DW}}$ between each step [Fig. 3(b) inset] [41,42]. When averaged over all domains and traveling directions, one can write $h(q, N) \sim \exp \left[-\left(q R N^{\alpha}\right)^{2}\right]$, assuming a constant displacement $R$ of superdomain boundaries during each step [40].

Here we used $R \sim 0.8 a$, the center of the PDF of domain boundary displacements for a single jump, where $a$ is the lattice parameter of square ASL [43]. The exponent $\alpha$ describes the nature of the particle motion and ranges from 0 to 1 . Two regimes, $\alpha<1 / 2$ and $\alpha>1 / 2$, correspond to subdiffusion and hyperdiffusion, respectively. There are two special cases: $\alpha=1$ describes unidirectional motion over the decorrelation time of the system, commonly referred to as "ballistic" motion, and $\alpha=1 / 2$ describes Brownian motion. In Figs. 3(b) and 3(c), we plot the temperature dependence of $\tau_{\mathrm{DW}}$ and $\alpha$ obtained by individually fitting $F(q, \tau)$ with Eq. (2) for fixed $q$. Here, the initial decay in $F(q, \tau)$, fit to delay times of $\approx 2 \mathrm{~s}$, provides insight into the block-spin collective dynamics. Our analysis does not eliminate the possible existence of faster $(\tau<0.1 \mathrm{sec})$ or slower dynamics occurring beyond the initial decay.

In Fig. 3(c), the exponent $\alpha$ starts off close to 0.65 at $335 \mathrm{~K}$ and drops to 0.5 as temperature approaches to $T_{N}$, suggesting that the nature of superdomain motion changes from ballistic to diffusive. This can be explained considering two types of domain boundaries: superdomain walls separating two AFM superdomains or phase boundaries separating AFM superdomains and paramagnetic regions. Consider, for example, an initially AFM-ordered ground state that encompasses the whole sample. When a superdomain wall spontaneously nucleates, the system tries to minimize the energy by pushing the superdomain wall out of the sample. The superdomain wall travels until it is scattered by another wall, is pinned by a defect, or reaches the sample's edge. Therefore, at low temperatures, superdomain walls appear to behave ballistically. On the other hand, at high temperatures, the sample is broken into small AFM superdomains separated by paramagnetic regions. In this regime, each AFM superdomain can move independently with no additional energy cost, and therefore the phase boundaries exhibit diffusive motion. This interpretation is also consistent with a continuous phase transition in which boundary effects lead to phase separation [43].

The characteristic time cost for domain wall motion $\tau_{\mathrm{DW}}$ increases at low temperatures and diverges as superdomain walls freeze at a singularity, as shown in Fig. 3(b). This type of behavior is often described using the VogelFulcher-Tammann (VFT) law in systems dominated by domains $[44,45]$ :

$$
\tau_{\mathrm{DW}}=\tau_{o} \exp \left[D T_{o} /(T-T o)\right],
$$

where $T_{o}$ is the freezing-in temperature and $D$ is the fragility of the system. The smaller the "fragility," the more the system deviates from an Arrhenius-type behavior.

The decay time, $\tau_{\mathrm{DW}}$ in Eq. (3), is well fitted using $\tau_{o}=0.003(2) \mathrm{s}, D=0.17(6)$, and $T_{o}=326(2) \mathrm{K}$ [solid line in Fig. 3(b)], indicating that the superdomain wall movement exponentially slows as $T$ approaches $T_{o}$ [46]. The value of $D$ obtained is surprisingly similar to that of magnetic domains of a spiral antiferromagnet $(D=0.14$ [47]). Our $\tau_{o}$, the characteristic fluctuation time as $T \rightarrow \infty$, is large compared to values observed for nanoparticles $\left(\approx 10^{-10}\right.$ s) [48]. This is consistent with the nature of superdomain boundary fluctuations that necessarily require multiple block-spin flips. If we consider individual blockspin flips in the limit that $T \gg T_{o}$, we find $\tau_{\mathrm{DW}}$ is well modeled by fluctuations involving approximately 4 block spins. (See Supplemental Material [16], Sec. S6.) This result is consistent with AFM domains fluctuating by one lattice unit cell when surrounded by PM regions.

Finally, we compare our random walk model to a stretched exponential function: $F(\tau)=a \exp \left[-\left(\tau / \tau_{F}\right)^{\gamma}\right]+$ $(1-a)$ that is commonly employed to understand XPCS data for collective phenomena in glasses and jammed systems $[44,45,47] . \tau_{F}$ and $\gamma$ are the decay constant and stretched exponent, respectively, while $(1-a)$ accounts for the finite, temperature dependent offset explained earlier. Figure 4 compares the temperature dependence of $\tau_{F}$ obtained from the stretched exponential model to $\tau_{\mathrm{DW}}$ obtained from the random walk model. A VFT fit of the form in Eq. (3) (solid lines) found that both models yield $D$ and $T_{o}$ values within the range of expected error. The ratio of $\tau_{o}$ from the stretched exponential fit to $\tau_{o}$ from the CTRW model is $\sim 20$, comparable to the total number of lattice units across the sample. This suggests that $\tau_{F}$ is related to the travel time of a superdomain boundary (taking approximately $20 \tau_{\mathrm{DW}}$ to move out of the sample). In addition, the exponent $\gamma$ decreases from 1.8 at 


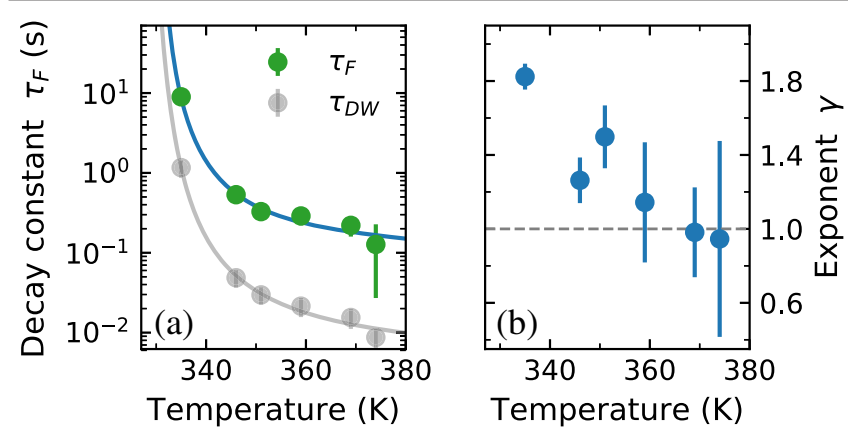

FIG. 4. (a) Temperature dependence of decay time $\tau_{F}$, compared with $\tau_{\mathrm{DW}}$. (b) Compressed exponent $\gamma$.

$T=335 \mathrm{~K}$ to $\approx 1$ as the sample temperature approaches $T_{N}$ [Fig. 4(b)]. Our random walk model therefore gives a natural explanation for $\gamma$ where a compressed $(\gamma>1)$ and a simple $(\gamma=1)$ exponential indicate collective and diffusive motion of superdomain boundaries, respectively.

In summary, resonant coherent $\mathrm{x}$-ray scattering provides unique insights for understanding the equilibrium behavior of a square ASL near its AFM-to-PM phase transition temperature $T_{N}$. As temperature decreases below $T_{N}$, AFM superdomain sizes increase and magnetic fluctuations slow. Applying both CTRW and the stretched exponential models to the time correlation of the AFM speckle pattern revealed a dynamical crossover temperature below $T_{N}$ near which superdomain wall motion changes from diffusive to ballistic. Below this crossover temperature, the superdomain walls exponentially slow down with decreasing temperature and freeze in at $T_{o}$ as determined by the VFT model.

These results show that superdomain-wall nucleation, annihilation, and motion are important for governing the complex equilibrium fluctuations of square artificial spin lattices. The methods described here can be readily applied to studies of the effects of disorder and defects in various artificial lattices [49-53]. Similar collective motion of spins likely exists in other phase separated materials and could be explored using coherent $\mathrm{x}$ rays [47,54]. Moreover, our findings concerning equilibrium fluctuations may prove important when engineering ASL for information technology or other applications [26-29,55].

The work is supported by the U.S. Department of Energy, Office of Science, Office of Basic Energy Sciences under Award No. DE-SC0016519. This research used resources of the Advanced Light Source, which is a DOE Office of Science User Facility under Contract No. DE-AC02-05CH11231. This research used resources 23-ID-1 beam line of the National Synchrotron Light Source II, a U.S. Department of Energy (DOE) Office of Science User Facility operated for the DOE Office of Science by Brookhaven National Laboratory under Contract No. DE-SC0012704. Work at the Molecular Foundry was supported by the Office of Science, Office of Basic Energy Sciences, of the U.S. Department of
Energy under Contract No. DE-AC02-05CH11231. Use of the Center for Nanoscale Materials, an Office of Science user facility, was supported by the U.S. Department of Energy, Office of Science, Office of Basic Energy Sciences, under Contract No. DE-AC02-06CH11357. This work was performed in part at the University of Kentucky Center for Nanoscale Science and Engineering and Center for Advanced Materials, members of the National Nanotechnology Coordinated Infrastructure (NNCI), which is supported by the National Science Foundation (ECCS-1542164). I. K. R was supported by EPSRC under Grant No. EP/I022562/1.

\author{
*xmchen@lbl.gov \\ †sroy@1bl.gov \\ todd.hastings@uky.edu
}

[1] R. Sibille, E. Lhotel, M. C. Hatnean, G. J. Nilsen, G. Ehlers, A. Cervellino, E. Ressouche, M. Frontzek, O. Zaharko, V. Pomjakushin et al., Nat. Commun. 8, 892 (2017).

[2] L. Balents, Nature (London) 464, 199 (2010).

[3] D. J. P. Morris, D. Tennant, S. Grigera, B. Klemke, C. Castelnovo, R. Moessner, C. Czternasty, M. Meissner, K. Rule, J.-U. Hoffmann et al., Science 326, 411 (2009).

[4] E. Mengotti, L. J. Heyderman, A. F. Rodríguez, F. Nolting, R. V. Hügli, and H.-B. Braun, Nat. Phys. 7, 68 (2011).

[5] J. P. Morgan, A. Stein, S. Langridge, and C. H. Marrows, Nat. Phys. 7, 75 (2011).

[6] Y.-L. Wang, Z.-L. Xiao, A. Snezhko, J. Xu, L. E. Ocola, R. Divan, J. E. Pearson, G. W. Crabtree, and W.-K. Kwok, Science 352, 962 (2016).

[7] E. Östman, H. Stopfel, I.-A. Chioar, U. B. Arnalds, A. Stein, V. Kapaklis, and B. Hjörvarsson, Nat. Phys. 14, 375 (2018).

[8] P. E. Lammert, V. H. Crespi, and C. Nisoli, New J. Phys. 14, 045009 (2012).

[9] G. Möller and R. Moessner, Phys. Rev. Lett. 96, 237202 (2006).

[10] C. Nisoli, R. Wang, J. Li, W. F. McConville, P. E. Lammert, P. Schiffer, and V. H. Crespi, Phys. Rev. Lett. 98, 217203 (2007).

[11] V. Kapaklis, U. B. Arnalds, A. Harman-Clarke, E. T. Papaioannou, M. Karimipour, P. Korelis, A. Taroni, P. C. Holdsworth, S. T. Bramwell, and B. Hjörvarsson, New J. Phys. 14, 035009 (2012).

[12] A. Farhan, P. M. Derlet, A. Kleibert, A. Balan, R. V. Chopdekar, M. Wyss, J. Perron, A. Scholl, F. Nolting, and L. J. Heyderman, Phys. Rev. Lett. 111, 057204 (2013).

[13] V. Kapaklis, U. B. Arnalds, A. Farhan, R. V. Chopdekar, A. Balan, A. Scholl, L. J. Heyderman, and B. Hjörvarsson, Nat. Nanotechnol. 9, 514 (2014).

[14] M. S. Andersson, S. D. Pappas, H. Stopfel, E. Östman, A. Stein, P. Nordblad, R. Mathieu, B. Hjörvarsson, and V. Kapaklis, Sci. Rep. 637097 (2016).

[15] For an example of superdomains in a different magnetic thin film system please see X. K. Hu, S. Sievers, A. Müller, V. Janke, and H. W. Schumacher, Phys. Rev. B 84, 024404 (2011).

[16] See Supplemental Material at http://link.aps.org/ supplemental/10.1103/PhysRevLett.123.197202 for the 
energy dependence of the AFM Bragg peak intensity in Sec. S1, the temperature history dependence of the AFM Bragg peak intensity $[14,17]$ in Sec. S2, the one-time correlation $g_{2}$, for high temperature datasets in Sec. S3, the construction of the CTRW model for superdomain walls [18-21] in Sec. S4, and the displacement of superdomain boundaries during each step used in the CTRW model in Sec. S5.

[17] D. Levis, L. F. Cugliandolo, L. Foini, and M. Tarzia, Phys. Rev. Lett. 110, 207206 (2013).

[18] C. Caronna, Y. Chushkin, A. Madsen, and A. Cupane, Phys. Rev. Lett. 100, 055702 (2008).

[19] J. Nelson, Phys. Rev. B 59, 15374 (1999).

[20] H. Guo, G. Bourret, M. K. Corbierre, S. Rucareanu, R. B. Lennox, K. Laaziri, L. Piche, M. Sutton, J. L. Harden, and R. L. Leheny, Phys. Rev. Lett. 102, 075702 (2009).

[21] L. Cipelletti, S. Manley, R. C. Ball, and D. A. Weitz, Phys. Rev. Lett. 84, 2275 (2000).

[22] S. Zhang, I. Gilbert, C. Nisoli, G.-W. Chern, M. J. Erickson, L. OBrien, C. Leighton, P. E. Lammert, V. H. Crespi, and P. Schiffer, Nature (London) 500, 553 (2013).

[23] J. Drisko, T. Marsh, and J. Cumings, Nat. Commun. 8, 14009 (2017).

[24] I. Gilbert, Y. Lao, I. Carrasquillo, L. OBrien, J. D. Watts, M. Manno, C. Leighton, A. Scholl, C. Nisoli, and P. Schiffer, Nat. Phys. 12, 162 (2016).

[25] We note that PEEM can be conducted at approximately 1 image per second when only a single polarization is used to detect changes in magnetization as described by A. Farhan, P. M. Derlet, A. Kleibert, A. Balan, R. V. Chopdekar, M. Wyss, L. Anghinolfi, F. Nolting, and L. J. Heyderman, Nat. Phys. 9, 375 (2013); Stroboscopic PEEM can also measure faster, repeatable dynamics such as switching in an applied field but cannot measure spontaneous fluctuations. See, for example, Z. Gu, M. E. Nowakowski, D. B. Carlton, R. Storz, M.-Y. Im, J. Hong, W. Chao, B. Lambson, P. Bennett, M. T. Alam, M. A. Marcus, A. Doran, A. Young, A. Scholl, P. Fischer, and J. Bokor, Nat. Commun. 6, 6466 (2015).

[26] J. H. Jensen, E. Folven, and G. Tufte, in Artificial Life Conference Proceedings (MIT Press, Cambridge, MA, 2018), pp. 15-22.

[27] H. Arava, N. Leo, D. Schildknecht, J. Cui, J. Vijayakumar, P. M. Derlet, A. Kleibert, and L. J. Heyderman, Phys. Rev. Applied 11, 054086 (2019).

[28] H. Arava, P. M. Derlet, J. Vijayakumar, J. Cui, N. S. Bingham, A. Kleibert, and L. J. Heyderman, Nanotechnology 29, 265205 (2018).

[29] M. Hehn, F. Montaigne, D. Lacour, Y. Perrin, B. Canals, N. Rougemaille, J. Grollier, D. Querlioz, and A. Masseboeuf, in Spintronics XI Vol. 10732 (SPIE Publications, 2018), p. 107323D, https://doi.org/10.1117/12.2325724.

[30] D. Doering, Y.-D. Chuang, N. Andresen, K. Chow, D. Contarato, C. Cummings, E. Domning, J. Joseph, J. Pepper, B. Smith et al., Rev. Sci. Instrum. 82, 073303 (2011).

[31] Y.-L. Xie, Z.-Z. Du, Z.-B. Yan, and J.-M. Liu, Sci. Rep. 5, 15875 (2015).

[32] See, for example, D. P. Landau, and K. Binder, A Guide to Monte Carlo Simulations in Statistical Physics, 2nd ed.
(Cambridge University Press, Cambridge, New York, 2005), which provides a pedagogical explanation about the finitesize scaling in Sec. IV.2.3.

[33] D. Attwood and A. Sakdinawat, X-rays and Extreme Ultraviolet Radiation: Principles and Applications (Cambridge University Press, Cambridge, England, 2017).

[34] I. Robinson, T. A. Assefa, Y. Cao, G. Gu, R. Harder, E. Maxey, and M. P. M. Dean, J. Supercond. Novel Magn. 32, 1 (2019).

[35] A single superdomain is equivalent to a "negative" pinhole, which will create an Airy ringlike diffraction pattern. The peak we see is, in fact, the Airy ring center, whose width doubles a normal speckle size (or the Airy ring fringe width).

[36] S. A. Morley, D. Alba Venero, J. M. Porro, S. T. Riley, A. Stein, P. Steadman, R. L. Stamps, S. Langridge, and C. H. Marrows, Phys. Rev. B 95, 104422 (2017).

[37] The speckle contrast $\beta$ depends only on x-ray coherence properties, scattering geometry, and x-ray penetration depth.

[38] X. M. Chen, V. Thampy, C. Mazzoli, A. M. Barbour, H. Miao, G. D. Gu, Y. Cao, J. M. Tranquada, M. P. M. Dean, and S. B. Wilkins, Phys. Rev. Lett. 117, 167001 (2016).

[39] E. W. Montroll and G. H. Weiss, J. Math. Phys. (N.Y.) 6, 167 (1965).

[40] A. Duri and L. Cipelletti, Europhys. Lett. 76, 972 (2006).

[41] R. Metzler and J. Klafter, Phys. Rep. 339, 1 (2000).

[42] J.-P. Bouchaud and A. Georges, Phys. Rep. 195, 127 (1990).

[43] L. F. Cugliandolo, J. Stat. Phys. 167, 499 (2017).

[44] G. Tarjus, S. A. Kivelson, Z. Nussinov, and P. Viot, J. Phys. Condens. Matter 17, R1143 (2005).

[45] F. Mallamace, C. Branca, C. Corsaro, N. Leone, J. Spooren, S.-H. Chen, and H. E. Stanley, Proc. Natl. Acad. Sci. U.S.A. 107, 22457 (2010).

[46] We note here that $T_{o}$ should not be confused with the blocking temperature of isolated block spins, which is at much lower temperature.

[47] S.-W. Chen, H. Guo, K. A. Seu, K. Dumesnil, S. Roy, and S. K. Sinha, Phys. Rev. Lett. 110, 217201 (2013).

[48] W. F. Brown, IEEE Trans. Magn. 15, 1196 (1979).

[49] J. C. Gartside, D. M. Arroo, D. M. Burn, V. L. Bemmer, A. Moskalenko, L. F. Cohen, and W. R. Branford, Nat. Nanotechnol. 13, 53 (2018).

[50] L. Savary and L. Balents, Phys. Rev. Lett. 118, 087203 (2017).

[51] O. Sendetskyi, L. Anghinolfi, V. Scagnoli, G. Möller, N. Leo, A. Alberca, J. Kohlbrecher, J. Lüning, U. Staub, and L. J. Heyderman, Phys. Rev. B 93, 224413 (2016).

[52] Y. Shen, O. Petrova, P. Mellado, S. Daunheimer, J. Cumings, and O. Tchernyshyov, New J. Phys. 14, 035022 (2012).

[53] A. Glavic, B. Summers, A. Dahal, J. Kline, W. Van Herck, A. Sukhov, A. Ernst, and D. K. Singh, Adv. Sci. 5, 1700856 (2018).

[54] J. Tao, K. Sun, W.-G. Yin, L. Wu, H. Xin, J. Wen, W. Luo, S. Pennycook, J. Tranquada, and Y. Zhu, Sci. Rep. 6, 37624 (2016).

[55] P. Neumann, R. Kolesov, B. Naydenov, J. Beck, F. Rempp, M. Steiner, V. Jacques, G. Balasubramanian, M. Markham, D. Twitchen et al., Nat. Phys. 6, 249 (2010). 